\title{
The phase diagram of neutral quark matter: Self-consistent treatment of quark masses
}

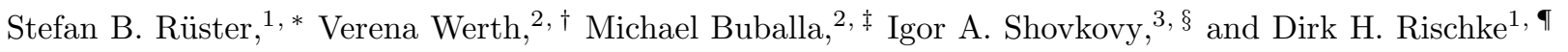 \\ ${ }^{1}$ Institut für Theoretische Physik, J.W. Goethe-Universität, D-60438 Frankfurt am Main, Germany \\ ${ }^{2}$ Institut für Kernphysik, Technische Universität Darmstadt, D-64289 Darmstadt, Germany \\ ${ }^{3}$ Frankfurt Institute for Advanced Studies, J.W. Goethe-Universität, D-60438 Frankfurt am Main, Germany
}

(Dated: October 22, 2018)

\begin{abstract}
We study the phase diagram of dense, locally neutral three-flavor quark matter within the framework of the Nambu-Jona-Lasinio model. In the analysis, dynamically generated quark masses are taken into account self-consistently. The phase diagram in the plane of temperature and quark chemical potential is presented. The results for two qualitatively different regimes, intermediate and strong diquark coupling strength, are presented. It is shown that the role of gapless phases diminishes with increasing diquark coupling strength.
\end{abstract}

\section{INTRODUCTION}

Theoretical studies suggest that baryon matter at sufficiently high density and sufficiently low temperature is a color superconductor. (For reviews on color superconductivity see, for example, Ref. [1].) In Nature, the highest densities of matter are reached in central regions of compact stars. There, the density might be as large as $10 \rho_{0}$ where $\rho_{0} \approx 0.15 \mathrm{fm}^{-3}$ is the saturation density. It is possible that baryonic matter is deconfined under such conditions and, perhaps, it is color-superconducting.

In compact stars, matter in the bulk is neutral with respect to the electric and color charges. Matter should also remain in $\beta$ equilibrium. Taking these constraints consistently into account may have a strong effect on the competition between different phases of deconfined quark matter at large baryon densities 2, 3, 4, 5, 6]. In this paper, we study this competition within the framework of a Nambu-Jona-Lasinio (NJL) model. The results are summarized in the phase diagram in the plane of temperature $(T)$ and quark chemical potential $(\mu)$.

The first attempt to obtain the phase diagram of dense, locally neutral three-flavor quark matter as a function of the strange quark mass, the quark chemical potential, and the temperature was made in Ref. 7]. It was shown that, at zero temperature and small values of the strange quark mass, the ground state of matter corresponds to the color-flavor-locked (CFL) phase [8, 9]. At some critical value of the strange quark mass, this is replaced by the gapless CFL (gCFL) phase [6]. In addition, several other phases were found at nonzero temperature. For instance, it was shown that there should exist a metallic CFL (mCFL) phase, a so-called uSC phase [10], as well

\footnotetext{
*Electronic address: ruester@th.physik.uni-frankfurt.de

$\dagger$ Electronic address: verena.werth@physik.tu-darmstadt.de

‡Electronic address: michael.buballa@physik.tu-darmstadt.de

$\S$ Electronic address: shovkovy@th.physik.uni-frankfurt.de on leave from Bogolyubov Institute for Theoretical Physics, 03143, Kiev, Ukraine

๑ Electronic address: drischke@th.physik.uni-frankfurt.de
}

as the standard two-flavor color-superconducting (2SC) phase [11, 12] and the gapless 2SC (g2SC) phase [5].

In Ref. 7], the effect of the strange quark mass was incorporated only approximately through a shift of the chemical potential of strange quarks, $\mu_{s} \rightarrow \mu_{s}-m_{s}^{2} /(2 \mu)$. While such an approach is certainly reliable at small values of the strange quark mass, it becomes uncontrollable with increasing the mass. The phase diagram of Ref. 7] was further developed in Refs. [13, 14] where the shiftapproximation in dealing with the strange quark was not employed any more. So far, however, quark masses were treated as free parameters, rather than dynamically generated quantities. In this paper, we study the phase diagram of dense, locally neutral three-flavor quark matter within the NJL model, treating dynamically generated quark masses self-consistently. Some results within this approach at zero temperature were also obtained in Refs. [3, 15].

As in Refs. 7, 13, 14], we restrict our analysis to locally neutral phases only. This automatically excludes, for example, mixed [16] and crystalline 17] phases. Taking them into account requires a special treatment which is outside the scope of this paper.

This paper is organized as follows. In Sec. II we introduce the model and, within this model, derive a complete set of gap equations and charge neutrality conditions. The numerical results for the phase diagrams in the plane of temperature and quark chemical potential in two qualitatively different regimes are presented in Sec. III Finally, our results are summarized in Sec. IV The Appendix contains some useful formulas.

\section{MODEL AND FORMALISM}

In this paper, we use a three-flavor quark model with a local NJL-type interaction. The Lagrangian density is 
given by

$$
\begin{aligned}
\mathcal{L} & =\bar{\psi}(i \not \partial-\hat{m}) \psi+G_{S} \sum_{a=0}^{8}\left[\left(\bar{\psi} \lambda_{a} \psi\right)^{2}+\left(\bar{\psi} i \gamma_{5} \lambda_{a} \psi\right)^{2}\right] \\
& +G_{D} \sum_{\gamma, c}\left[\bar{\psi}_{\alpha}^{a} i \gamma_{5} \epsilon^{\alpha \beta \gamma} \epsilon_{a b c}\left(\psi_{C}\right)_{\beta}^{b}\right]\left[\left(\bar{\psi}_{C}\right)_{\rho}^{r} i \gamma_{5} \epsilon^{\rho \sigma \gamma} \epsilon_{r s c} \psi_{\sigma}^{s}\right] \\
& -K\left\{\operatorname{det}_{f}\left[\bar{\psi}\left(1+\gamma_{5}\right) \psi\right]+\operatorname{det}_{f}\left[\bar{\psi}\left(1-\gamma_{5}\right) \psi\right]\right\},
\end{aligned}
$$

where the quark spinor field $\psi_{\alpha}^{a}$ carries color $(a=r, g, b)$ and flavor $(\alpha=u, d, s)$ indices. The matrix of quark current masses is given by $\hat{m}=\operatorname{diag}_{f}\left(m_{u}, m_{d}, m_{s}\right)$. Regarding other notations, $\lambda_{a}$ with $a=1, \ldots, 8$ are the Gell-Mann matrices in flavor space, and $\lambda_{0} \equiv \sqrt{2 / 3} \mathbb{1}_{f}$. The charge conjugate spinors are defined as follows: $\psi_{C}=C \bar{\psi}^{T}$ and $\bar{\psi}_{C}=\psi^{T} C$, where $\bar{\psi}=\psi^{\dagger} \gamma^{0}$ is the Dirac conjugate spinor and $C=i \gamma^{2} \gamma^{0}$ is the charge conjugation matrix.

The model in Eq. (11) should be viewed as an effective model of strongly interacting matter that captures at least some key features of QCD dynamics. The Lagrangian density contains three different interaction terms which are chosen to respect the symmetries of QCD. Note that we include the 't Hooft interaction whose strength is determined by the coupling constant $K$. This term breaks $U(1)$ axial symmetry.

The term in the second line of Eq. (11) describes a scalar diquark interaction in the color antitriplet and flavor antitriplet channel. For symmetry reasons there should also be a pseudoscalar diquark interaction with the same coupling constant. This term would be important to describe Goldstone boson condensation in the CFL phase [18]. In this paper, however, we neglect this possibility and therefore drop the pseudoscalar diquark term.

We use the following set of model parameters [19]:

$$
\begin{aligned}
m_{u, d} & =5.5 \mathrm{MeV} \\
m_{s} & =140.7 \mathrm{MeV} \\
G_{S} \Lambda^{2} & =1.835 \\
K \Lambda^{5} & =12.36 \\
\Lambda & =602.3 \mathrm{MeV} .
\end{aligned}
$$

After fixing the masses of the up and down quarks at equal values, $m_{u, d}=5.5 \mathrm{MeV}$, the other four parameters are chosen to reproduce the following four observables of vacuum QCD [19]: $m_{\pi}=135.0 \mathrm{MeV}, m_{K}=497.7 \mathrm{MeV}$, $m_{\eta^{\prime}}=957.8 \mathrm{MeV}$, and $f_{\pi}=92.4 \mathrm{MeV}$. This parameter set gives $m_{\eta}=514.8 \mathrm{MeV}[19]$.

In Ref. [19], the diquark coupling $G_{D}$ was not fixed by the fit of the meson spectrum in vacuum. In general, it is expected to be of the same order as the quark-antiquark coupling $G_{S}$. In this paper, we study in detail two possible cases: the regime of intermediate coupling strength with $G_{D}=\frac{3}{4} G_{S}$, and the regime of strong coupling with $G_{D}=G_{S}$. The comparison of phase diagrams in these two cases will turn out to be very instructive.
The grand partition function, up to an irrelevant normalization constant, is given by

$$
\mathcal{Z} \equiv \mathrm{e}^{-\Omega V / T}=\int \mathcal{D} \bar{\psi} \mathcal{D} \psi \mathrm{e}^{i \int_{X}\left(\mathcal{L}+\bar{\psi} \hat{\mu} \gamma^{0} \psi\right)},
$$

where $\Omega$ is the thermodynamic potential density, $V$ is the volume of the three-space, and $\hat{\mu}$ is a diagonal matrix of quark chemical potentials. In chemical equilibrium (which provides $\beta$ equilibrium as a special case), the nontrivial components of this matrix are extracted from the following relation:

$\mu_{a b}^{\alpha \beta}=\left(\mu \delta^{\alpha \beta}+\mu_{Q} Q_{f}^{\alpha \beta}\right) \delta_{a b}+\left[\mu_{3}\left(T_{3}\right)_{a b}+\mu_{8}\left(T_{8}\right)_{a b}\right] \delta^{\alpha \beta}$.

Here $\mu$ is the quark chemical potential (by definition, $\mu=\mu_{B} / 3$ where $\mu_{B}$ is the baryon chemical potential), $\mu_{Q}$ is the chemical potential of electric charge, while $\mu_{3}$ and $\mu_{8}$ are color chemical potentials associated with two mutually commuting color charges of the $S U(3)_{c}$ gauge group. The explicit form of the electric charge matrix is $Q_{f}=\operatorname{diag}_{f}\left(\frac{2}{3},-\frac{1}{3},-\frac{1}{3}\right)$, and the explicit form of the color charge matrices is $T_{3}=\operatorname{diag}_{c}\left(\frac{1}{2},-\frac{1}{2}, 0\right)$ and $\sqrt{3} T_{8}=$ $\operatorname{diag}_{c}\left(\frac{1}{2}, \frac{1}{2},-1\right)$.

In order to calculate the mean-field thermodynamic potential at temperature $T$, we first linearize the interaction in the presence of the diquark condensates $\Delta_{c} \sim\left(\bar{\psi}_{C}\right)_{\alpha}^{a} i \gamma_{5} \epsilon^{\alpha \beta c} \epsilon_{a b c} \psi_{\beta}^{b}$ (no sum over $c$ ) and the quarkantiquark condensates $\sigma_{\alpha} \sim \bar{\psi}_{\alpha}^{a} \psi_{\alpha}^{a}$ (no sum over $\alpha$ ). Then, integrating out the quark fields and neglecting the fluctuations of composite order parameters, we arrive at the following expression for the thermodynamic potential:

$$
\begin{aligned}
\Omega & =\Omega_{L}+\frac{1}{4 G_{D}} \sum_{c=1}^{3}\left|\Delta_{c}\right|^{2}+2 G_{S} \sum_{\alpha=1}^{3} \sigma_{\alpha}^{2} \\
& -4 K \sigma_{u} \sigma_{d} \sigma_{s}-\frac{T}{2 V} \sum_{K} \ln \operatorname{det} \frac{S^{-1}}{T},
\end{aligned}
$$

where we also added the contribution of leptons, $\Omega_{L}$, which will be specified later.

We should note that we have restricted ourselves to field contractions corresponding to the Hartree approximation. In a more complete treatment, among others, the 't Hooft interaction term gives also rise to mixed contributions containing both diquark and quark-antiquark condensates, i.e., $\propto \sum_{\alpha=1}^{3} \sigma_{\alpha}\left|\Delta_{\alpha}\right|^{2}[20]$. In this study, as in Refs. 3, 21], we neglect such terms for simplicity. While their presence may change the results quantitatively, one does not expect them to modify the qualitative structure of the phase diagram.

In Eq. (5), $S^{-1}$ is the inverse full quark propagator in the Nambu-Gorkov representation,

$$
S^{-1}=\left(\begin{array}{cc}
{\left[G_{0}^{+}\right]^{-1}} & \Phi^{-} \\
\Phi^{+} & {\left[G_{0}^{-}\right]^{-1}}
\end{array}\right)
$$


with the diagonal elements being the inverse Dirac propagators of quarks and of charge-conjugate quarks,

$$
\left[G_{0}^{ \pm}\right]^{-1}=\gamma^{\mu} K_{\mu} \pm \hat{\mu} \gamma_{0}-\hat{M}
$$

where $K^{\mu}=\left(k_{0}, \mathbf{k}\right)$ denotes the four-momentum of the quark. At nonzero temperature, we use the Matsubara imaginary time formalism. Therefore, the energy $k_{0}$ is replaced with $-i \omega_{n}$ where $\omega_{n} \equiv(2 n+1) \pi T$ are the fermionic Matsubara frequencies. Accordingly, the sum over $K$ in Eq. (5) should be interpreted as a sum over integer $n$ and an integral over the three-momentum $\mathbf{k}$.

The constituent quark mass matrix is defined as $\hat{M}=$ $\operatorname{diag}_{f}\left(M_{u}, M_{d}, M_{s}\right)$ with

$$
M_{\alpha}=m_{\alpha}-4 G_{S} \sigma_{\alpha}+2 K \sigma_{\beta} \sigma_{\gamma},
$$

where $\sigma_{\alpha}$ are the quark-antiquark condensates, and the set of indices $(\alpha, \beta, \gamma)$ is a permutation of $(u, d, s)$.

The off-diagonal components of the propagator (6) are the so-called gap matrices given in terms of three diquark condensates. The color-flavor structure of these matrices is given by

$$
\left(\Phi^{-}\right)_{a b}^{\alpha \beta}=-\sum_{c} \epsilon^{\alpha \beta c} \epsilon_{a b c} \Delta_{c} \gamma_{5}
$$

and $\Phi^{+}=\gamma^{0}\left(\Phi^{-}\right)^{\dagger} \gamma^{0}$. Here, as before, $a$ and $b$ refer to the color components and $\alpha$ and $\beta$ refer to the flavor components. Hence, the gap parameters $\Delta_{1}, \Delta_{2}$, and $\Delta_{3}$ correspond to the down-strange, the up-strange and the updown diquark condensates, respectively. All three of them originate from the color-antitriplet, flavor-antitriplet diquark pairing channel. For simplicity, the color and flavor symmetric condensates are neglected in this study. They were shown to be small and not crucial for the qualitative understanding of the phase diagram [7].

By making use of the results in Appendix $\mathrm{A}$ the determinant of the inverse quark propagator can be decomposed as follows:

$$
\operatorname{det} \frac{S^{-1}}{T}=\prod_{i=1}^{18}\left(\frac{\omega_{n}^{2}+\epsilon_{i}^{2}}{T^{2}}\right)^{2},
$$

where $\epsilon_{i}$ are eighteen independent positive energy eigenvalues. The Matsubara summation in Eq. (5) can then be done analytically by employing the relation [22]

$$
\sum_{n} \ln \left(\frac{\omega_{n}^{2}+\epsilon_{i}^{2}}{T^{2}}\right)=\frac{\left|\epsilon_{i}\right|}{T}+2 \ln \left(1+\mathrm{e}^{-\frac{\left|\epsilon_{i}\right|}{T}}\right) .
$$

Then, we arrive at the following mean-field expression for the pressure $(p \equiv-\Omega)$ :

$$
\begin{aligned}
p & =\frac{1}{2 \pi^{2}} \sum_{i=1}^{18} \int_{0}^{\Lambda} \mathrm{d} k k^{2}\left[\left|\epsilon_{i}\right|+2 T \ln \left(1+\mathrm{e}^{-\frac{\left|\epsilon_{i}\right|}{T}}\right)\right] \\
& +4 K \sigma_{u} \sigma_{d} \sigma_{s}-\frac{1}{4 G_{D}} \sum_{c=1}^{3}\left|\Delta_{c}\right|^{2}-2 G_{S} \sum_{\alpha=1}^{3} \sigma_{\alpha}^{2} \\
& +\frac{T}{\pi^{2}} \sum_{l=e, \mu} \sum_{\epsilon= \pm} \int_{0}^{\infty} \mathrm{d} k k^{2} \ln \left(1+\mathrm{e}^{-\frac{E_{l}-\epsilon \mu_{l}}{T}}\right),
\end{aligned}
$$

where the contribution of electrons and muons with masses $m_{e} \approx 0.511 \mathrm{MeV}$ and $m_{\mu} \approx 105.66 \mathrm{MeV}$ were included. Note that muons may exist in matter in $\beta$ equilibrium and, therefore, they are included in the model for consistency. However, being about 200 times heavier than electrons, they do not play a big role in the analysis.

In this paper, we assume that there are no trapped neutrinos in quark matter. This is expected to be a good approximation for matter inside a neutron star after the short deleptonization period is over. The effect of neutrino trapping will be addressed elsewhere [23].

The expression for the pressure in Eq. (12) has a physical meaning only when the chiral and color superconducting order parameters, $\sigma_{\alpha}$ and $\Delta_{c}$, satisfy the following set of six gap equations:

$$
\begin{aligned}
\frac{\partial p}{\partial \sigma_{\alpha}} & =0, \\
\frac{\partial p}{\partial \Delta_{c}} & =0 .
\end{aligned}
$$

To enforce the conditions of local charge neutrality in dense matter, we also require three other equations to be satisfied,

$$
\begin{gathered}
n_{Q} \equiv \frac{\partial p}{\partial \mu_{Q}}=0, \\
n_{3} \equiv \frac{\partial p}{\partial \mu_{3}}=0, \\
n_{8} \equiv \frac{\partial p}{\partial \mu_{8}}=0 .
\end{gathered}
$$

These fix the values of the three corresponding chemical potentials, $\mu_{Q}, \mu_{3}$ and $\mu_{8}$. After these are fixed, only the quark chemical potential $\mu$ is left as a free parameter.

\section{RESULTS}

In order to obtain the phase diagram, we have to find the ground state of matter for each given set of the parameters in the model. In the case of locally neutral matter, there are two parameters that should be specified: temperature $T$ and quark chemical potential $\mu$. After these are fixed, one has to compare the values of the pressure in all competing neutral phases of quark matter. The ground state corresponds to the phase with the highest pressure.

Before calculating the pressure, given by Eq. (12), one has to find the values of the chiral and the color superconducting order parameters, $\sigma_{\alpha}$ and $\Delta_{c}$, as well as the values of the three charge chemical potentials, $\mu_{Q}, \mu_{3}$ and $\mu_{8}$. These are obtained by solving the coupled set of six gap equations (13) together with the three neutrality conditions (14). By using standard numerical recipes, it is not extremely difficult to find a solution to the given set of nine nonlinear equations. Complications arise, however, due to the fact that often the solution is not unique.

The existence of different solutions to the same set of equations, (13) and (14), reflects the physical fact that 
there could exist several competing neutral phases with different physical properties. Among these phases, all but one are unstable or metastable. In order to take this into account in our study, we look for the solutions of the following 8 types:

1. Normal quark (NQ) phase: $\Delta_{1}=\Delta_{2}=\Delta_{3}=0$;

2. 2SC phase: $\Delta_{1}=\Delta_{2}=0$, and $\Delta_{3} \neq 0$;

3. 2SCus phase: $\Delta_{1}=\Delta_{3}=0$, and $\Delta_{2} \neq 0$;

4. 2SCds phase: $\Delta_{2}=\Delta_{3}=0$, and $\Delta_{1} \neq 0$;

5. uSC phase: $\Delta_{2} \neq 0, \Delta_{3} \neq 0$, and $\Delta_{1}=0$;

6. dSC phase: $\Delta_{1} \neq 0, \Delta_{3} \neq 0$, and $\Delta_{2}=0$;

7. $\mathrm{sSC}$ phase: $\Delta_{1} \neq 0, \Delta_{2} \neq 0$, and $\Delta_{3}=0$;

8. CFL phase: $\Delta_{1} \neq 0, \Delta_{2} \neq 0, \Delta_{3} \neq 0$.

Then, we calculate the values of the pressure in all nonequivalent phases, and determine the ground state as the phase with the highest pressure. After this is done, we study additionally the spectrum of low-energy quasiparticles in search for the existence of gapless modes. This allows us to refine the specific nature of the ground state.

In the above definition of the eight phases in terms of $\Delta_{c}$, we have ignored the quark-antiquark condensates $\sigma_{\alpha}$. In fact, in the chiral limit $\left(m_{\alpha}=0\right)$, the quantities $\sigma_{\alpha}$ are good order parameters and we could define additional sub-phases characterized by nonvanishing values of one or more $\sigma_{\alpha}$. With the model parameters at hand, however, chiral symmetry is broken explicitly by the nonzero current quark masses, and the values of $\sigma_{\alpha}$ never vanish. Hence, in a strict sense it is impossible to define any new phases in terms of $\sigma_{\alpha}$.

Of course, this does not exclude the possibility of discontinuous changes in $\sigma_{\alpha}$ at some line in the plane of temperature and quark chemical potential, thereby constituting a first-order phase transition line. It is generally expected that the "would-be" chiral phase transition remains first-order at low temperatures, even for nonzero quark masses. Above some critical temperature, however, this line could end in a critical endpoint and there is only a smooth crossover at higher temperatures. Among others, this picture emerges from NJL-model studies, both, without [24] and with [25] diquark pairing (see also Ref. 21]). We should therefore expect a similar behavior in our analysis.

Our numerical results for neutral quark matter are summarized in Figs. 1 and 2 These are the phase diagrams in the plane of temperature and quark chemical potential, obtained in the mean-field approximation in model (1) in the case of an intermediate diquark coupling strength, $G_{D}=\frac{3}{4} G_{S}$, and in the case of a strong coupling, $G_{D}=G_{S}$, respectively. The corresponding dynamical quark masses, gap parameters, and three charge chemical potentials are displayed in Figs. 3 and 4 respectively. All quantities are plotted as functions of $\mu$ for three different fixed values of the temperature: $T=0,20,40 \mathrm{MeV}$ in the case of $G_{D}=\frac{3}{4} G_{S}$ (see Fig. 3) and $T=0,40,60 \mathrm{MeV}$ in the case of $G_{D}=G_{S}$ (see Fig. 4).

Let us begin with the results in the case of the diquark coupling being $G_{D}=\frac{3}{4} G_{S}$. In the region of small quark chemical potentials and low temperatures, the phase diagram is dominated by the normal phase in which the approximate chiral symmetry is broken, and in which quarks have relatively large constituent masses. This is denoted by $\chi \mathrm{SB}$ in Fig. 1] With increasing the temperature, this phase changes smoothly into the NQ phase in which quark masses are relatively small. Because of explicit breaking of the chiral symmetry in the model at hand, there is no need for a phase transition between the two regimes.

However, as pointed out above, the symmetry argument does not exclude the possibility of an "accidental" (first-order) chiral phase transition. As expected, at lower temperatures we find a line of first-order chiral phase transitions. It is located within a relatively narrow window of the quark chemical potentials $(336 \mathrm{MeV} \lesssim \mu \lesssim$ $368 \mathrm{MeV}$ ) which are of the order of the vacuum values of the light-quark constituent masses. (For the parameters used in our calculations one obtains $M_{u}=M_{d}=$ $367.7 \mathrm{MeV}$ and $M_{s}=549.5 \mathrm{MeV}$ in vacuum [19].) At this critical line, the quark chiral condensates, as well as the quark constituent masses, change discontinuously. With increasing temperature, the size of the discontinuity decreases, and the line terminates at the endpoint located at $\left(T_{\mathrm{cr}}, \mu_{\mathrm{cr}}\right) \approx(56,336) \mathrm{MeV}$, see Fig. 1.

The location of the critical endpoint is consistent with other mean field studies of NJL models with similar sets of parameters [21, 24, 25]. This agreement does not need to be exact because, in contrast to the studies in Refs. 21, 24, 25], here we imposed the condition of electric charge neutrality in quark matter. (Note that the color neutrality is satisfied automatically in the normal phase.) One may argue, however, that the additional constraint of neutrality is unlikely to play a big role in the vicinity of the endpoint.

It is appropriate to mention here that the location of the critical endpoint might be affected very much by fluctuations of the composite chiral fields. These are not included in the mean-field studies of the NJL model. In fact, this is probably the main reason for their inability to pin down the location of the critical endpoint consistent, for example, with lattice calculations [26]. (It is fair to mention that the current lattice calculations are not very reliable at nonzero $\mu$ either.) Therefore, the predictions of this study, as well as of those in Refs. 21, 24, 25], regarding the critical endpoint cannot be considered as very reliable.

When the quark chemical potential exceeds some critical value and the temperature is not too large, a Cooper instability with respect to diquark condensation should develop in the system. Without enforcing neutrality, i.e., if the chemical potentials of up and down quarks are 
equal, this happens immediately after the chiral phase transition when the density becomes nonzero [25]. In the present model, this is not the case at low temperatures.

In order to understand this, let us inspect the various quantities at $T=0$ which are displayed in the upper three panels of Fig. 3. At the chiral phase boundary, the up and down quark masses become relatively small, whereas the strange quark mass experiences only a moderate drop of about $84 \mathrm{MeV}$ induced by the 't Hooft interaction. This is not sufficient to populate any strange quark states at the given chemical potential, and the system mainly consists of up and down quarks together with a small fraction of electrons, see Fig. 5. The electric charge chemical potential which is needed to maintain neutrality in this regime is between about -73 and $-94 \mathrm{MeV}$. It turns out that the resulting splitting of the up and down quark Fermi momenta is too large for the given diquark coupling strength to enable diquark pairing and the system stays in the normal phase.

At $\mu \approx 432 \mathrm{MeV}$, the chemical potential felt by the strange quarks, $\mu-\mu_{Q} / 3$, reaches the strange quark mass and the density of strange quarks becomes nonzero. At first, this density is too small to play a sizeable role in neutralizing matter, or in enabling strange-nonstrange cross-flavor diquark pairing, see Fig. 5. The NQ phase becomes metastable against the gapless CFL (gCFL) phase at $\mu_{\mathrm{gCFL}} \approx 443 \mathrm{MeV}$. This is the point of a first-order phase transition. It is marked by a drop of the strange quark mass by about $121 \mathrm{MeV}$. As a consequence, strange quarks become more abundant and pairing gets easier. Yet, in the gCFL phase, the strange quark mass is still relatively large, and the standard BCS pairing between strange and light (i.e., up and down) quarks is not possible. In contrast to the regular CFL phase, the gCFL phase requires a nonzero density of electrons to stay electrically neutral. At $T=0$, therefore, one could use the value of the electron density as a formal order parameter that distinguishes these two phases [6].

With increasing the chemical potential further (still at $T=0$ ), the strange quark mass decreases and the crossflavor Cooper pairing gets stronger. Thus, the gCFL phase eventually turns into the regular CFL phase at $\mu_{\mathrm{CFL}} \approx 457 \mathrm{MeV}$. The electron density goes to zero at this point, as it should. This is indicated by the vanishing value of $\mu_{Q}$ in the CFL phase, see the upper right panel in Fig. 3] We remind that the CFL phase is neutral because of having equal number densities of all three quark flavors, $n_{u}=n_{d}=n_{s}$, see Fig. [5] and 6] This equality is enforced by the pairing mechanism, and this is true even when the quark masses are not exactly equal [27].

Let us mention here that the same NJL model at zero temperature was studied previously in Ref. [3]. Our results agree qualitatively with those of Ref. 3] only when the quark chemical potential is larger than the critical value for the transition to the CFL phase at $457 \mathrm{MeV}$. The appearance of the gCFL phase for $443 \lesssim \mu \lesssim$ $457 \mathrm{MeV}$ was not recognized in Ref. [3], however. Instead, it was suggested that there exists a narrow (about
$12 \mathrm{MeV}$ wide) window of values of the quark chemical potential around $\mu \approx 450 \mathrm{MeV}$ in which the $2 \mathrm{SC}$ phase is the ground state. By carefully checking the same region, we find that the 2SC phase does not appear there.

This is illustrated in Fig. 7 where the pressure of three different solutions is displayed. Had we ignored the gCFL solution (thin solid line), the 2SC solution (dashed line) would indeed be the most favored one in the interval between $\mu \approx 445 \mathrm{MeV}$ and $\mu \approx 457 \mathrm{MeV}$. After including the gCFL phase in the analysis, this is no longer the case.

Now let us turn to the case of nonzero temperature. One might suggest that this should be analogous to the zero temperature case, except that Cooper pairing is somewhat suppressed by thermal effects. In contrast to this naive expectation, the thermal distributions of quasiparticles together with the local neutrality conditions open qualitatively new possibilities that were absent at $T=0$. As in the case of the two-flavor model of Ref. [5], a moderate thermal smearing of mismatched Fermi surfaces could increase the probability of creating zero-momentum Cooper pairs without running into a conflict with Pauli blocking. This leads to the appearance of several stable color-superconducting phases that could not exist at zero temperatures.

With increasing the temperature, the first qualitatively new feature in the phase diagram appears when $5 \lesssim T \lesssim 10 \mathrm{MeV}$. In this temperature interval, the NQ phase is replaced by the uSC phase when the quark chemical potential exceeds the critical value of about $444 \mathrm{MeV}$. The corresponding transition is a first-order phase transition, see Fig. 11 Increasing the chemical potential further by several $\mathrm{MeV}$, the uSC phase is then replaced by the gCFL phase, and the gCFL phase later turns gradually into the $(\mathrm{m}) \mathrm{CFL}$ phase. (In this study, we do not distinguish between the CFL phase and the mCFL phase 7.). Note that, in the model at hand, the transition between the uSC and the gCFL phase is of second order in the following two temperature intervals: $5 \lesssim T \lesssim 9 \mathrm{MeV}$ and $T \gtrsim 24 \mathrm{MeV}$. On the other hand, it is a first-order transition when $9 \lesssim T \lesssim 24 \mathrm{MeV}$. Leaving aside its unusual appearance, this is likely to be an "accidental" property in the model for a given set of parameters. For a larger value of the diquark coupling, in particular, such a feature does not appear, see Fig. 2

The transition from the gCFL to the CFL phase is a smooth crossover at all $T \neq 0$ [7, 13]. The reason is that the electron density is not a good order parameter that could be used to distinguish the gCFL from the CFL phase when the temperature is nonzero. This is also confirmed by our numerical results for the electric charge chemical potential $\mu_{Q}$ in Fig. 3. While at zero temperature the value of $\mu_{Q}$ vanishes identically in the CFL phase, this is not the case at finite temperatures.

Another new feature in the phase diagram appears when the temperature is above about $11 \mathrm{MeV}$. In this case, with increasing the quark chemical potential, the Cooper instability happens immediately after the $\chi \mathrm{SB}$ phase. The corresponding critical value of the quark che- 
mical potential is rather low, about $365 \mathrm{MeV}$. The first color superconducting phase is the gapless 2SC (g2SC) phase [5]. This phase is replaced with the $2 \mathrm{SC}$ phase in a crossover transition only when $\mu \gtrsim 445 \mathrm{MeV}$. The $2 \mathrm{SC}$ is then followed by the gapless uSC (guSC) phase, by the uSC phase, by the gCFL phase and, eventually, by the CFL phase (see Fig. 11).

In the NJL model at hand, determined by the parameters in Eq. (2), we do not find the dSC phase as the ground state anywhere in the phase diagram. This is similar to the conclusion of Refs. 7, 14], but differs from that of Refs. 10, 13]. This should not be surprising because, as was noted earlier [14], the appearence of the dSC phase is rather sensitive to a specific choice of parameters in the NJL model.

The phase diagram in Fig. 1has a very specific ordering of quark phases. One might ask if this ordering is robust against the modification of the parameters of the model at hand. Below we argue that some features are indeed quite robust, while others are not.

It should be clear that the appearance of colorsuperconducting phases under the stress of neutrality constraints is very sensitive to the strength of diquark coupling. In the case of two-flavor quark matter, this was demonstrated very clearly in Ref. [5] at zero as well as at nonzero temperatures. Similar conclusions were also reached in the study of three-flavor quark matter at zero temperature [15].

In the model at hand, it is instructive to study the phase diagram in the regime of strong diquark coupling, $G_{D}=G_{S}$. The corresponding results are summarized in the diagram in Fig. 2 As we see, the main qualitative difference between the diagrams in Figs. 1 and 2 occurs at intermediate values of the quark chemical potential. While at $G_{D}=\frac{3}{4} G_{S}$, there is a large region of the g2SC phase sandwiched between the low-temperature and high-temperature NQ phases, this is not the case at stronger coupling, $G_{D}=G_{S}$.

The last observation can easily be explained by the fact that with increasing diquark coupling strength, the condensation energy also increases and therefore Cooper pairing is favorable, even if there is a larger mismatch of the Fermi surfaces due to charge neutrality constraints. Moreover, in the presence of large gaps, the Fermi surfaces are smeared over a region of order $\Delta$. Therefore additional thermal smearing is of no further help, and it is not surprising that the thermal effects in a model with sufficiently strong coupling are qualitatively the same as in models without neutrality constraints imposed: thermal fluctuations can only destroy the pairing. In the model with a not very strong coupling, on the other hand, the interplay of the charge neutrality and thermal fluctuations is more subtle. The normal phase of cold quark matter develops a Cooper instability and becomes a color superconductor only after a moderate thermal smearing of the quark Fermi surfaces is introduced [5].

Other than this, the qualitative features of the phase diagrams in Figs. 1 and 2 are similar. Of course, in the case of the stronger coupling, the critical lines lie systematically at higher values of the temperature and at lower values of the quark chemical potential. In this context one should note that the first-order phase boundary between the two normal regimes " $\chi \mathrm{SB}$ " and "NQ" is insensitive to the diquark coupling. Therefore, upon increasing $G_{D}$ it stays at its place until it is eventually "eaten" up by the expanding $2 \mathrm{SC}$ phase. As a result, there is no longer a critical endpoint in Fig. 2 but only a critical point where the first-order normal $(\chi \mathrm{SB})-2 \mathrm{SC}$ phase boundary changes into second order.

\section{CONCLUSIONS}

In this paper, we studied the $T-\mu$ phase diagram of neutral three-flavor quark matter within the NJL model of Ref. [19] in which the chiral symmetry is broken explicitly by small but nonzero current quark masses. As in the previous studies 7, 13, 14], we use the mean-field approximation in the analysis. In contrast to Refs. [7, 13, 14], in this paper the constituent quark masses are treated selfconsistently as dynamically generated quantities. The main results are summarized in Figs. 1] and 2

By comparing our results with those in Ref. 7] (see Fig. 11b there), we notice several important differences. First of all, we observe that a self-consistent treatment of quark masses strongly influences the competition between different quark phases. As was noticed earlier in Ref. [25], there exists a subtle interplay between the two main effects. On the one hand, the actual values of the quark masses directly influence the competition between different normal and color-superconducting phases. On the other hand, competing phases themselves determine the magnitude of the masses. Very often, this leads to first-order phase transitions, in which certain regions in the mass-parameter space become inaccessible.

Some differences to the results in Ref. [7] are related to a different choice of model parameters. Most importantly, the value of the diquark coupling $G_{D}=\frac{3}{4} G_{S}$ is considerably weaker than in the NJL model of Ref. [7]. This can be easily seen by comparing the magnitude of the zero-temperature gap at a given value of the quark chemical potential, say at $\mu=500 \mathrm{MeV}$, in the two models. It is $\Delta_{0}^{(500)} \approx 76 \mathrm{MeV}$ in this paper, and it is $\Delta_{0}^{(500)} \approx 140 \mathrm{MeV}$ in Ref. 7]. (Note that the strength of the diquark pairing in Ref. [13] is even weaker, corresponding to $\Delta_{0}^{(500)} \approx 20 \mathrm{MeV}$.) It should be noted that even the case of the strong coupling, $G_{D}=G_{S}$, which corresponds to $\Delta_{0}^{(500)} \approx 120 \mathrm{MeV}$, is still slightly weaker than that in Ref. [7]. In this case, however, the corresponding results differ mostly because the quark masses are treated very differently.

Because of the weaker diquark coupling strength, the Cooper instabilities in Fig. 10 happen systematically at higher values of the quark chemical potential than in Ref. 7]. In particular, this is most clearly seen from the 
critical lines of the transition to the (g)CFL phase. Another consequence of the weaker interaction is the possibility of a thermal enhancement of the (g)2SC Cooper pairing at intermediate values of the quark chemical potential. This kind of enhancement was studied in detail in Ref. [5]. Making use of the same arguments, one can tell immediately how the phase diagram in Fig. 11 should change with increasing or decreasing the diquark coupling strength.

In particular, with increasing (decreasing) the diquark coupling strength, the region of the $(\mathrm{g}) 2 \mathrm{SC}$ phase at intermediate values of the quark chemical potential should expand (shrink) along the temperature direction. The regions covered by the other (i.e., uSC and CFL) phases should have qualitatively the same shape, but shift to lower (higher) values of the quark chemical potential and to higher (lower) values of the temperature. In the case of strong coupling, in particular, these general arguments are confirmed by our numerical calculations. The corresponding phase diagram is shown in Fig. 2

Several comments are in order regarding the choice of the NJL model used here. The model is defined by the set of parameters in Eq. (2) which were fitted to reproduce several important QCD properties in vacuum [19]. (Note that the same model was also used in Ref. 3].) It is expected, therefore, that this is a reasonable effective model of QCD that captures the main features of both chiral and color-superconducting pairing dynamics. Also, a relatively small value of the cutoff parameter in the model, see Eq. (2e), should not necessarily be viewed as a bad feature of the model. In fact, this might simply mimic a natural property of the full theory in which the coupling strength of relevant interactions is quenched at large momenta.

In this relation, note that the approach of Ref. [13] regarding the cutoff parameter in the NJL model is very different. It is said there that a large value of this parameter is beneficial in order to extract results which are insensitive to a specific choice of the cutoff. However, we do not find any physical argument that would support this requirement. Instead, we insist on having an effective model that describes reasonably well the QCD properties at zero quark chemical potential. We do not pretend, of course, that a naive extrapolation of the model to large densities can be rigorously justified. In absence of a better alternative, however, this seems to be the only sensible choice.

The results of this paper might be relevant for understanding the physics of (hybrid) neutron stars with quark cores, in which the deleptonization is completed. In order to obtain a phase diagram that could be applied to protoneutron stars, one has to generalize the analysis to take into account neutrino trapping. This work is in progress now [23].

In the end, it might be appropriate to mention that, despite the progress in our understanding of the phase diagram of neutral dense quark matter, there still exists a fundamental problem here. The reason is that some regions of the phase diagrams in Figs. 1 and 2 correspond to phases that are known to be unstable [28]. Of course, it is of prime importance to resolve this issue.

Note added. While writing our paper, we learned that a partially overlapping study is being done by D. Blaschke, S. Fredriksson, H. Grigorian, A.M. Öztaş, and F. Sandin [29].

\section{Acknowledgments}

This work was supported in part by the Virtual Institute of the Helmholtz Association under grant No. VH-VI-041 and by Gesellschaft für Schwerionenforschung (GSI) and by Bundesministerium für Bildung und Forschung (BMBF). S. R. thanks for using the Center for Scientific Computing (CSC) of the Johann Wolfgang Goethe-Universität Frankfurt am Main.

\section{APPENDIX A: EVALUATION OF THE THERMODYNAMIC POTENTIAL}

We use the following ordering of the quark field components:

$$
\psi=\left(\psi_{u}^{r}, \psi_{d}^{r}, \psi_{s}^{r}, \psi_{u}^{g}, \psi_{d}^{g}, \psi_{s}^{g}, \psi_{u}^{b}, \psi_{d}^{b}, \psi_{s}^{b}\right)^{T}
$$

In this basis, the matrices of quark current and constituent masses read

$$
\hat{m}=\operatorname{diag}\left(m_{u}, m_{d}, m_{s}, m_{u}, m_{d}, m_{s}, m_{u}, m_{d}, m_{s}\right)
$$

and

$$
\hat{M}=\operatorname{diag}\left(M_{u}, M_{d}, M_{s}, M_{u}, M_{d}, M_{s}, M_{u}, M_{d}, M_{s}\right),
$$

respectively, with $M_{\alpha}$ given by Eq. (8). Moreover, the matrix of quark chemical potentials takes the general form

$$
\hat{\mu}=\operatorname{diag}\left(\mu_{u}^{r}, \mu_{d}^{r}, \mu_{s}^{r}, \mu_{u}^{g}, \mu_{d}^{g}, \mu_{s}^{g}, \mu_{u}^{b}, \mu_{d}^{b}, \mu_{s}^{b}\right) .
$$

Finally, the explicit color-flavor structure of the gap matrices $\Phi^{ \pm}$[see Eq. (9)] is given by

$$
\Phi^{-}=-\gamma_{5}\left(\begin{array}{ccccccccc}
0 & 0 & 0 & 0 & \Delta_{3} & 0 & 0 & 0 & \Delta_{2} \\
0 & 0 & 0 & -\Delta_{3} & 0 & 0 & 0 & 0 & 0 \\
0 & 0 & 0 & 0 & 0 & 0 & -\Delta_{2} & 0 & 0 \\
0 & -\Delta_{3} & 0 & 0 & 0 & 0 & 0 & 0 & 0 \\
\Delta_{3} & 0 & 0 & 0 & 0 & 0 & 0 & 0 & \Delta_{1} \\
0 & 0 & 0 & 0 & 0 & 0 & 0 & -\Delta_{1} & 0 \\
0 & 0 & -\Delta_{2} & 0 & 0 & 0 & 0 & 0 & 0 \\
0 & 0 & 0 & 0 & 0 & -\Delta_{1} & 0 & 0 & 0 \\
\Delta_{2} & 0 & 0 & 0 & \Delta_{1} & 0 & 0 & 0 & 0
\end{array}\right),
$$

and $\Phi^{+}=-\left(\Phi^{-}\right)^{\dagger}$. By making use of the symmetries in the model, we choose all three parameters $\Delta_{c}$ to be real. 
In order to calculate the last term in the thermodynamical potential in Eq. (5), it is useful to rewrite the determinant of the inverse full propagator as $\operatorname{det} S^{-1}=$ $\operatorname{det}\left(\gamma_{0} \gamma_{0} S^{-1}\right)=\operatorname{det}\left(\gamma_{0}\right) \operatorname{det}\left(\gamma_{0} S^{-1}\right)=\operatorname{det}\left(\gamma_{0} S^{-1}\right)$. One should also note that the matrices $\gamma_{0}\left[G_{0}^{ \pm}\right]^{-1}$ and $\gamma_{0} \Phi^{ \pm}$can be expressed in terms of the spin projectors,

$$
\begin{aligned}
\gamma_{0}\left[G_{0}^{ \pm}\right]^{-1} & =\sum_{s}\left(\begin{array}{cc}
k_{0} \pm \hat{\mu}-\hat{M} & -s k \\
-s k & k_{0} \pm \hat{\mu}+\hat{M}
\end{array}\right) \mathcal{P}_{s} \\
\gamma_{0} \Phi^{ \pm} & = \pm \sum_{s}\left(\begin{array}{cc}
0 & \hat{\Phi} \\
-\hat{\Phi} & 0
\end{array}\right) \mathcal{P}_{s}
\end{aligned}
$$

where the two projectors are defined as

$$
\mathcal{P}_{s}=\frac{1}{2}(1+s \boldsymbol{\sigma} \cdot \hat{\mathbf{k}}), \quad \text { for } \quad s= \pm .
$$

Here $\hat{\mathbf{k}} \equiv \mathbf{k} / k, k \equiv|\mathbf{k}|$, and $\hat{\Phi}$ represents only the color-flavor part of the gap matrix in Eq. (A5), i.e., $\Phi^{ \pm} \equiv \pm \gamma_{5} \hat{\Phi}$. By making use of the definition in Eq. (6), as well as Eqs. A6 and (A7), we obtain the following representation:

$$
\gamma_{0} S^{-1}=\sum_{s} \hat{S}_{s}^{-1} \mathcal{P}_{s}
$$

where $\hat{S}_{s}^{-1}=\mathbb{1} k_{0}-\mathcal{M}_{s}$, and

$$
\mathcal{M}_{s}=\left(\begin{array}{cccc}
-\hat{\mu}+\hat{M} & s k & 0 & \hat{\Phi} \\
s k & -\hat{\mu}-\hat{M} & -\hat{\Phi} & 0 \\
0 & -\hat{\Phi} & \hat{\mu}+\hat{M} & s k \\
\hat{\Phi} & 0 & s k & \hat{\mu}-\hat{M}
\end{array}\right)
$$

(with $s= \pm$ ) is real and symmetric. Since there is no explicit energy dependence in $\mathcal{M}_{s}$, their eigenvalues $\epsilon_{i}$ determine the quasiparticle dispersion relations, $k_{0}=\epsilon_{i}(k)$. By using the matrix relation $\ln \operatorname{det}(A)=\operatorname{Tr} \ln (A)$ as well as the properties of projectors $\mathcal{P}_{s}$, we derive

$$
\ln \operatorname{det}\left(\gamma_{0} S^{-1}\right)=\ln \left(\operatorname{det} \hat{S}_{+}^{-1} \cdot \operatorname{det} \hat{S}_{-}^{-1}\right) \text {. }
$$

It turns out that the two determinants appearing on the right hand side of this equation are equal, i.e., $\operatorname{det} \hat{S}_{-}^{-1}=$ $\operatorname{det} \hat{S}_{+}^{-1}$. From the physics viewpoint, this identity reflects the twofold spin degeneracy of the spectrum of quark quasiparticles. The formal proof of this degeneracy is straightforward after noticing that the following matrix relation is satisfied:

$$
\hat{S}_{-s}^{-1}=\mathcal{R} \hat{S}_{s}^{-1} \mathcal{R}^{-1}
$$

where

$$
\mathcal{R}=\left(\begin{array}{rrrr}
1 & 0 & 0 & 0 \\
0 & -1 & 0 & 0 \\
0 & 0 & -1 & 0 \\
0 & 0 & 0 & 1
\end{array}\right)
$$

is a unitary matrix with unit determinant, $\operatorname{det} \mathcal{R}=1$.
Another observation, which turns out to be helpful in the calculation, is that the determinant $\operatorname{det} \hat{S}_{s}^{-1}\left(k_{0}\right)$ is an even function of $k_{0}$, i.e., $\operatorname{det} \hat{S}_{s}^{-1}\left(-k_{0}\right)=\operatorname{det} \hat{S}_{s}^{-1}\left(k_{0}\right)$. This is a formal consequence of the following matrix relation:

$$
\hat{S}_{s}^{-1}\left(-k_{0}\right)=-\mathcal{B} \hat{S}_{s}^{-1}\left(k_{0}\right) \mathcal{B}^{-1}
$$

where the explicit form of the unitary matrix $\mathcal{B}$ is

$$
\mathcal{B}=\left(\begin{array}{rrrr}
0 & 0 & 0 & i \\
0 & 0 & -i & 0 \\
0 & i & 0 & 0 \\
-i & 0 & 0 & 0
\end{array}\right)
$$

It satisfies $\operatorname{det} \mathcal{B}=1$. The invariance of the determinant $\operatorname{det} \hat{S}_{s}^{-1}\left(k_{0}\right)$ with respect to the change of the energy sign, $k_{0} \rightarrow-k_{0}$, is directly related to the use of the NambuGorkov basis for quark fields. In this basis, for each quasiparticle excitation with a positive energy $k_{0}=\epsilon(k)$, there exists a corresponding excitation with a negative energy $k_{0}=-\epsilon(k)$. Therefore, the result for the determinant should read

$$
\operatorname{det}\left(S^{-1}\right)=\prod_{i=1}^{18}\left(k_{0}^{2}-\epsilon_{i}^{2}\right)^{2} .
$$

In order to simplify the numerical calculation of the eigenvalues of the matrix $\mathcal{M}_{+}$, defined in Eq. A10, we first write it in a block-diagonal form. The total dimension of this matrix is $36 \times 36$. With a proper ordering of its rows and columns, it decomposes into 6 diagonal blocks of dimension $4 \times 4$ and one diagonal block of dimension $12 \times 12$. The explicit form of these blocks reads

$\mathcal{M}_{+}^{(1)}=\left(\begin{array}{cccc}-\mu_{d}^{r}+M_{d} & k & 0 & -\Delta_{3} \\ k & -\mu_{d}^{r}-M_{d} & \Delta_{3} & 0 \\ 0 & \Delta_{3} & \mu_{u}^{g}+M_{u} & k \\ -\Delta_{3} & 0 & k & \mu_{u}^{g}-M_{u}\end{array}\right)$

$$
\mathcal{M}_{+}^{(2)}=\left(\begin{array}{cccc}
\mu_{d}^{r}-M_{d} & k & 0 & -\Delta_{3} \\
k & \mu_{d}^{r}+M_{d} & \Delta_{3} & 0 \\
0 & \Delta_{3} & -\mu_{u}^{g}-M_{u} & k \\
-\Delta_{3} & 0 & k & -\mu_{u}^{g}+M_{u}
\end{array}\right)
$$

$$
\mathcal{M}_{+}^{(3)}=\left(\begin{array}{cccc}
-\mu_{s}^{r}+M_{s} & k & 0 & -\Delta_{2} \\
k & -\mu_{s}^{r}-M_{s} & \Delta_{2} & 0 \\
0 & \Delta_{2} & \mu_{u}^{b}+M_{u} & k \\
-\Delta_{2} & 0 & k & \mu_{u}^{b}-M_{u}
\end{array}\right)
$$

$$
\mathcal{M}_{+}^{(4)}=\left(\begin{array}{cccc}
\mu_{s}^{r}-M_{s} & k & 0 & -\Delta_{2} \\
k & \mu_{s}^{r}+M_{s} & \Delta_{2} & 0 \\
0 & \Delta_{2} & -\mu_{u}^{b}-M_{u} & k \\
-\Delta_{2} & 0 & k & -\mu_{u}^{b}+M_{u}
\end{array}\right)
$$


and

$$
\begin{gathered}
\mathcal{M}_{+}^{(5)}=\left(\begin{array}{cccc}
-\mu_{s}^{g}+M_{s} & k & 0 & -\Delta_{1} \\
k & -\mu_{s}^{g}-M_{s} & \Delta_{1} & 0 \\
0 & \Delta_{1} & \mu_{d}^{b}+M_{d} & k \\
-\Delta_{1} & 0 & k & \mu_{d}^{b}-M_{d}
\end{array}\right), \\
\mathcal{M}_{+}^{(6)}=\left(\begin{array}{cccc}
\mu_{s}^{g}-M_{s} & k & 0 & -\Delta_{1} \\
k & \mu_{s}^{g}+M_{s} & \Delta_{1} & 0 \\
0 & \Delta_{1} & -\mu_{d}^{b}-M_{d} & k \\
-\Delta_{1} & 0 & k & -\mu_{d}^{b}+M_{d}
\end{array}\right),
\end{gathered}
$$

$$
\mathcal{M}_{+}^{(7)}=\left(\begin{array}{cccccccccccc}
-\mu_{u}^{r}-M_{u} & k & 0 & 0 & 0 & 0 & 0 & -\Delta_{3} & 0 & 0 & 0 & -\Delta_{2} \\
k & -\mu_{u}^{r}+M_{u} & 0 & 0 & 0 & 0 & \Delta_{3} & 0 & 0 & 0 & \Delta_{2} & 0 \\
0 & 0 & \mu_{u}^{r}-M_{u} & k & 0 & \Delta_{3} & 0 & 0 & 0 & \Delta_{2} & 0 & 0 \\
0 & 0 & k & \mu_{u}^{r}+M_{u} & -\Delta_{3} & 0 & 0 & 0 & -\Delta_{2} & 0 & 0 & 0 \\
0 & 0 & 0 & -\Delta_{3} & -\mu_{d}^{g}-M_{d} & k & 0 & 0 & 0 & 0 & 0 & -\Delta_{1} \\
0 & 0 & \Delta_{3} & 0 & k & -\mu_{d}^{g}+M_{d} & 0 & 0 & 0 & 0 & \Delta_{1} & 0 \\
0 & \Delta_{3} & 0 & 0 & 0 & 0 & \mu_{d}^{g}-M_{d} & k & 0 & \Delta_{1} & 0 & 0 \\
-\Delta_{3} & 0 & 0 & 0 & 0 & 0 & k & \mu_{d}^{g}+M_{d} & -\Delta_{1} & 0 & 0 & 0 \\
0 & 0 & 0 & -\Delta_{2} & 0 & 0 & 0 & -\Delta_{1} & -\mu_{s}^{b}-M_{s} & k & 0 & 0 \\
0 & 0 & \Delta_{2} & 0 & 0 & 0 & \Delta_{1} & 0 & k & -\mu_{s}^{b}+M_{s} & 0 & 0 \\
0 & \Delta_{2} & 0 & 0 & 0 & \Delta_{1} & 0 & 0 & 0 & 0 & \mu_{s}^{b}-M_{s} & k \\
-\Delta_{2} & 0 & 0 & 0 & -\Delta_{1} & 0 & 0 & 0 & 0 & 0 & k & \mu_{s}^{b}+M_{s}
\end{array}\right) .
$$

Out of 36 eigenvalues from all seven blocks, there are 18 positive and 18 negative eigenvalues. Out of total 18 positive eigenvalues, 9 of them correspond to quark type quasiparticles and the other 9 correspond to antiquark type quasiparticles. In our calculation, we extract all 36 eigenvalues numerically and then use them in the calculation of the pressure, see Eqs. (10) through (12).

Here, it might be interesting to note that the eigenvalues of the $4 \times 4$ matrices can be calculated analytically in the limit when two quark masses appearing in each of them are equal. For example, when $M_{d}=M_{u}$, the four eigenvalues of matrix $\mathcal{M}_{+}^{(1)}$ are given by

$$
\lambda_{i}^{(1)}= \pm \sqrt{\left(\frac{\mu_{d}^{r}+\mu_{u}^{g}}{2} \pm \sqrt{M_{u}^{2}+k^{2}}\right)^{2}+\Delta_{3}^{2}}-\frac{\mu_{d}^{r}-\mu_{u}^{g}}{2},
$$

while the eigenvalues of $\mathcal{M}_{+}^{(2)}$ differ only by the sign in front of the second term,

$$
\lambda_{i}^{(2)}= \pm \sqrt{\left(\frac{\mu_{d}^{r}+\mu_{u}^{g}}{2} \pm \sqrt{M_{u}^{2}+k^{2}}\right)^{2}+\Delta_{3}^{2}}+\frac{\mu_{d}^{r}-\mu_{u}^{g}}{2} .
$$

When the value of $\delta M \equiv M_{d}-M_{u}$ is nonzero but small, the corrections to the above eigenvalues are $\pm M_{u} \delta M /\left(2 \sqrt{M_{u}^{2}+k^{2}}\right)$ with the plus sign in the case of antiparticle modes, and the minus sign in the case of particle modes. The eigenvalues of $\mathcal{M}_{+}^{(3)}$ and $\mathcal{M}_{+}^{(4)}$ in the limit $M_{s}=M_{u}$, as well as the eigenvalues of $\mathcal{M}_{+}^{(5)}$ and $\mathcal{M}_{+}^{(6)}$ in the limit $M_{s}=M_{d}$, are similar.
[1] K. Rajagopal and F. Wilczek, hep-ph/0011333 M. Alford, Ann. Rev. Nucl. Part. Sci. 51, 131 (2001); T. Schäfer, hep-ph/0304281 D. H. Rischke, Prog. Part. Nucl. Phys. 52, 197 (2004); H.-C. Ren, hep-ph/0404074
M. Huang, hep-ph/0409167 I. A. Shovkovy, nucl-th/0410091

[2] M. Alford and K. Rajagopal, JHEP 0206, 031 (2002).

[3] A. W. Steiner, S. Reddy, and M. Prakash, Phys. Rev. D 
66, 094007 (2002).

[4] M. Huang, P. F. Zhuang and W. Q. Chao, Phys. Rev. D 67, 065015 (2003).

[5] I. Shovkovy and M. Huang, Phys. Lett. B 564, 205 (2003); M. Huang and I. Shovkovy, Nucl. Phys. A 729, 835 (2003).

[6] M. Alford, C. Kouvaris, and K. Rajagopal, Phys. Rev. Lett. 92, 222001 (2004); Phys. Rev. D 71, 034002 (2004).

[7] S. B. Rüster, I. A. Shovkovy and D. H. Rischke, Nucl. Phys. A 743, 127 (2004).

[8] M. G. Alford, K. Rajagopal, and F. Wilczek, Nucl. Phys. B537, 443 (1999).

[9] I. A. Shovkovy and L. C. R. Wijewardhana, Phys. Lett. B 470, 189 (1999); T. Schäfer, Nucl. Phys. B575, 269 (2000).

[10] K. Iida, T. Matsuura, M. Tachibana, and T. Hatsuda, Phys. Rev. Lett. 93, 132001 (2004).

[11] M. Alford, K. Rajagopal, and F. Wilczek, Phys. Lett. B 422, 247 (1998); R. Rapp, T. Schäfer, E. V. Shuryak, and M. Velkovsky, Phys. Rev. Lett. 81, 53 (1998).

[12] D. T. Son, Phys. Rev. D 59, 094019 (1999); T. Schäfer and F. Wilczek, Phys. Rev. D 60, 114033 (1999); D. K. Hong, V. A. Miransky, I. A. Shovkovy, and L. C. R. Wijewardhana, Phys. Rev. D 61, 056001 (2000); R. D. Pisarski and D. H. Rischke, Phys. Rev. D 61, 051501 (2000); W. E. Brown, J. T. Liu, and H.-C. Ren, Phys. Rev. D 61, 114012 (2000).

[13] K. Fukushima, C. Kouvaris and K. Rajagopal, Phys. Rev. D 71, 034002 (2005).

[14] I. A. Shovkovy, S. B. Rüster and D. H. Rischke, J. Phys. G 31, S849 (2005).

[15] H. Abuki, M. Kitazawa and T. Kunihiro, hep-ph/0412382

[16] F. Neumann, M. Buballa and M. Oertel, Nucl. Phys. A 714, 481 (2003); I. Shovkovy, M. Hanauske and M. Huang, Phys. Rev. D 67, 103004 (2003); S. Reddy and G. Rupak, Phys. Rev. C 71, 025201 (2005).

[17] M. G. Alford, J. A. Bowers and K. Rajagopal, Phys. Rev. D 63, 074016 (2001); R. Casalbuoni, R. Gatto, M. Mannarelli, and G. Nardulli, Phys. Rev. D 66, 014006 (2002); I. Giannakis, J. T. Liu, and H.-C. Ren, Phys. Rev. D 66, 031501 (2002).

[18] M. Buballa, Phys. Lett. B 609, 57 (2005); M. M. Forbes, hep-ph/0411001

[19] P. Rehberg, S. P. Klevansky and J. Hüfner, Phys. Rev. C 53, 410 (1996).

[20] R. Rapp, T. Schäfer, E. V. Shuryak and M. Velkovsky, Annals Phys. 280, 35 (2000).

[21] M. Buballa, Phys. Rep. 407, 205 (2005).

[22] J. I. Kapusta, Finite-temperature field theory, (University Press, Cambridge, 1989).

[23] S. B. Rüster, V. Werth, M. Buballa, I. A. Shovkovy, and D. H. Rischke, in preparation.

[24] M. Asakawa and K. Yazaki, Nucl. Phys. A 504, 668 (1989); Nucl. Phys. B 538, 215 (1999); O. Scavenius, A. Mocsy, I. N. Mishustin and D. H. Rischke, Phys. Rev. C 64, 045202 (2001).

[25] M. Buballa and M. Oertel, Nucl. Phys. A 703, 770 (2002).

[26] Z. Fodor and S. D. Katz, JHEP 0404, 050 (2004).

[27] K. Rajagopal and F. Wilczek, Phys. Rev. Lett. 86, 3492 (2001).

[28] M. Huang and I. A. Shovkovy, Phys. Rev. D 70, 051501(R) (2004); Phys. Rev. D 70, 094030 (2004); R. Casalbuoni, R. Gatto, M. Mannarelli, G. Nardulli and M. Ruggieri, Phys. Lett. B 605, 362 (2005); I. Giannakis and H.-C. Ren, Phys. Lett. B 611, 137 (2005); M. Alford and Q. H. Wang, J. Phys. G 31, 719 (2005).

[29] D. Blaschke, S. Fredriksson, H. Grigorian, A.M. Öztaş, and F. Sandin, hep-ph/0503194 


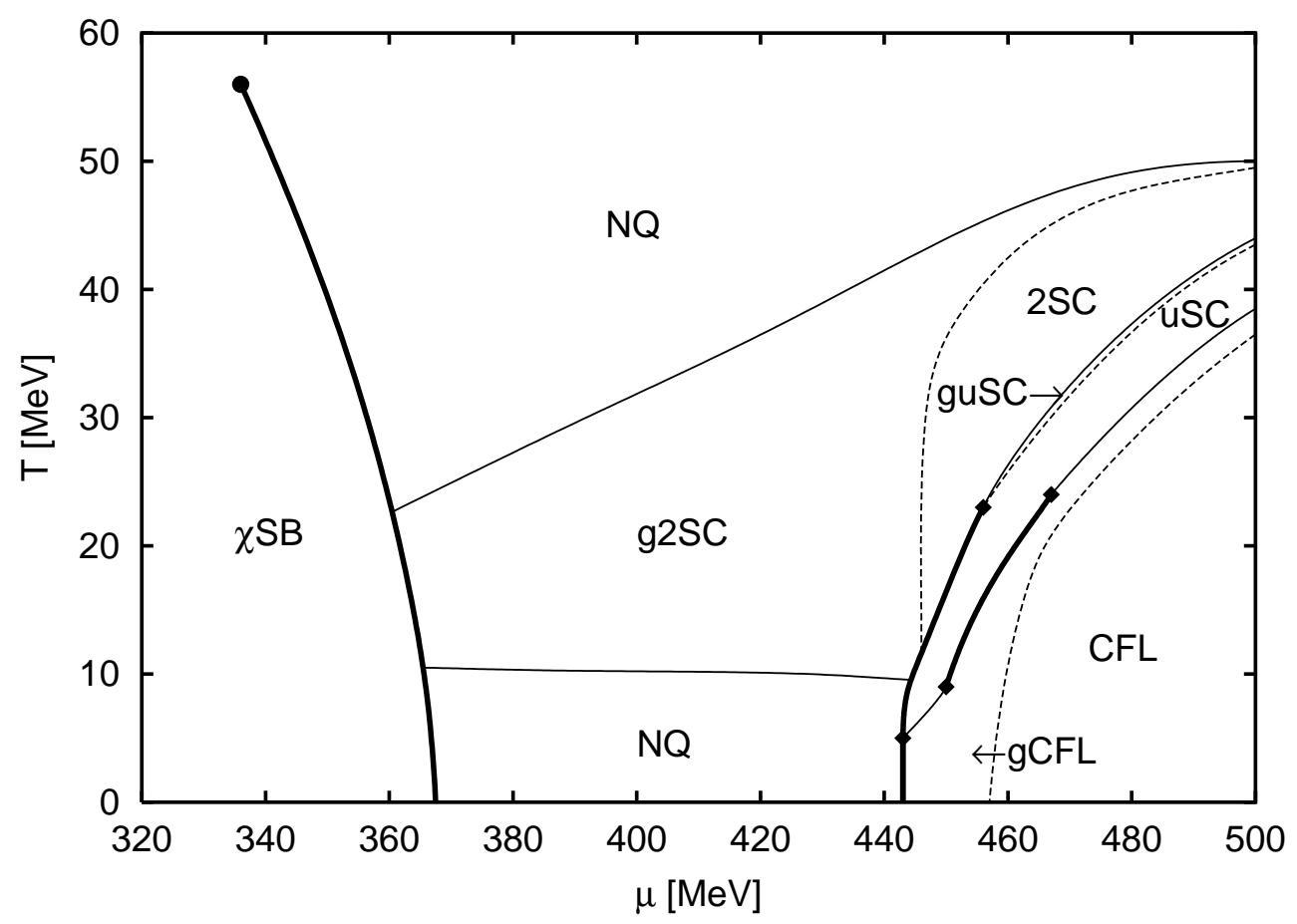

FIG. 1: The phase diagram of neutral quark matter in the regime of intermediate diquark coupling strength, $G_{D}=\frac{3}{4} G_{S}$. First-order phase boundaries are indicated by bold solid lines, whereas the thin solid lines mark second-order phase boundaries between two phases which differ by one or more nonzero diquark condensates. The dashed lines indicate the (dis-)appearance of gapless modes in different phases, and they do not correspond to phase transitions.

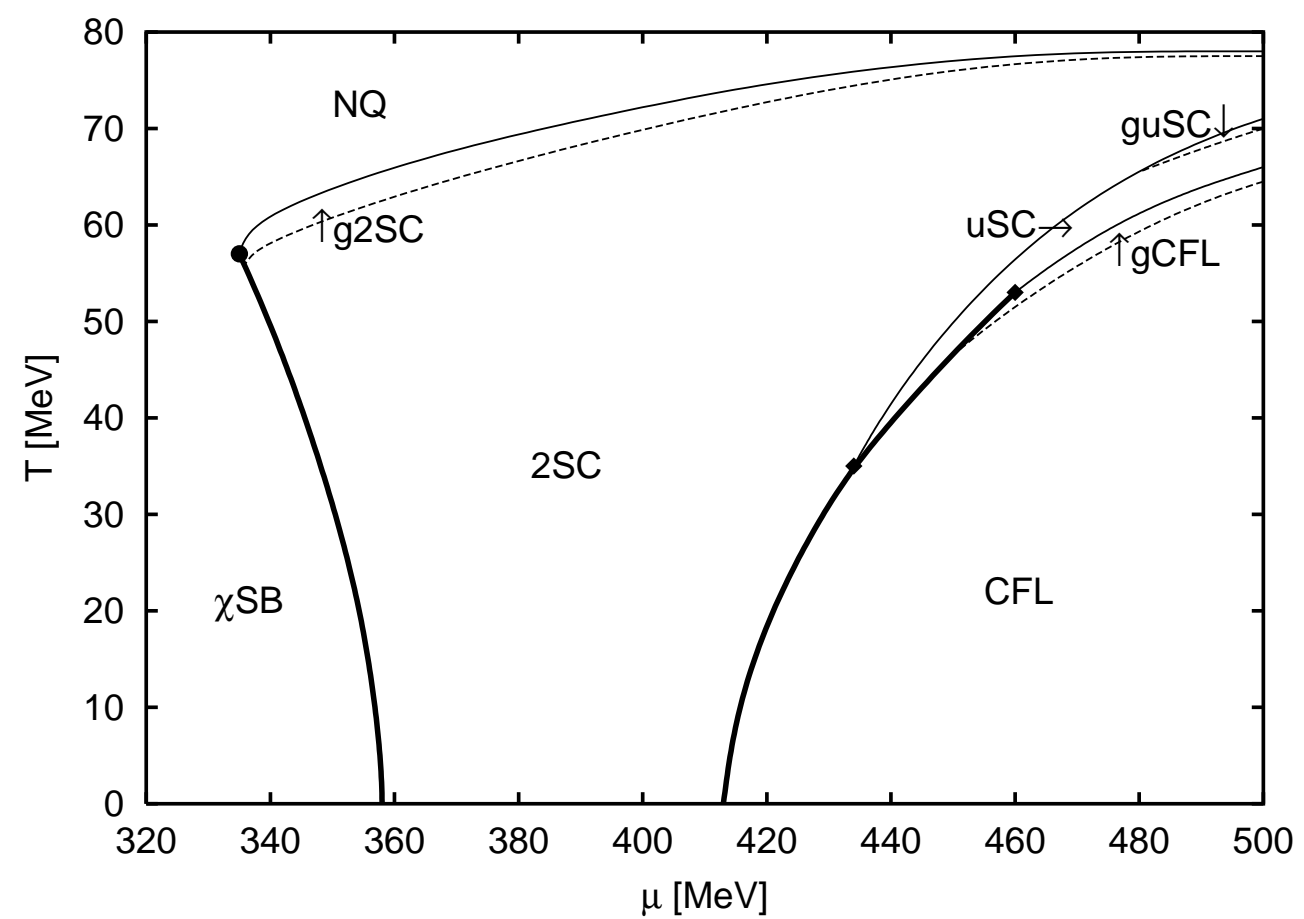

FIG. 2: The phase diagram of neutral quark matter in the regime of strong diquark coupling, $G_{D}=G_{S}$. The meaning of the various line types is the same as in Fig. 11 

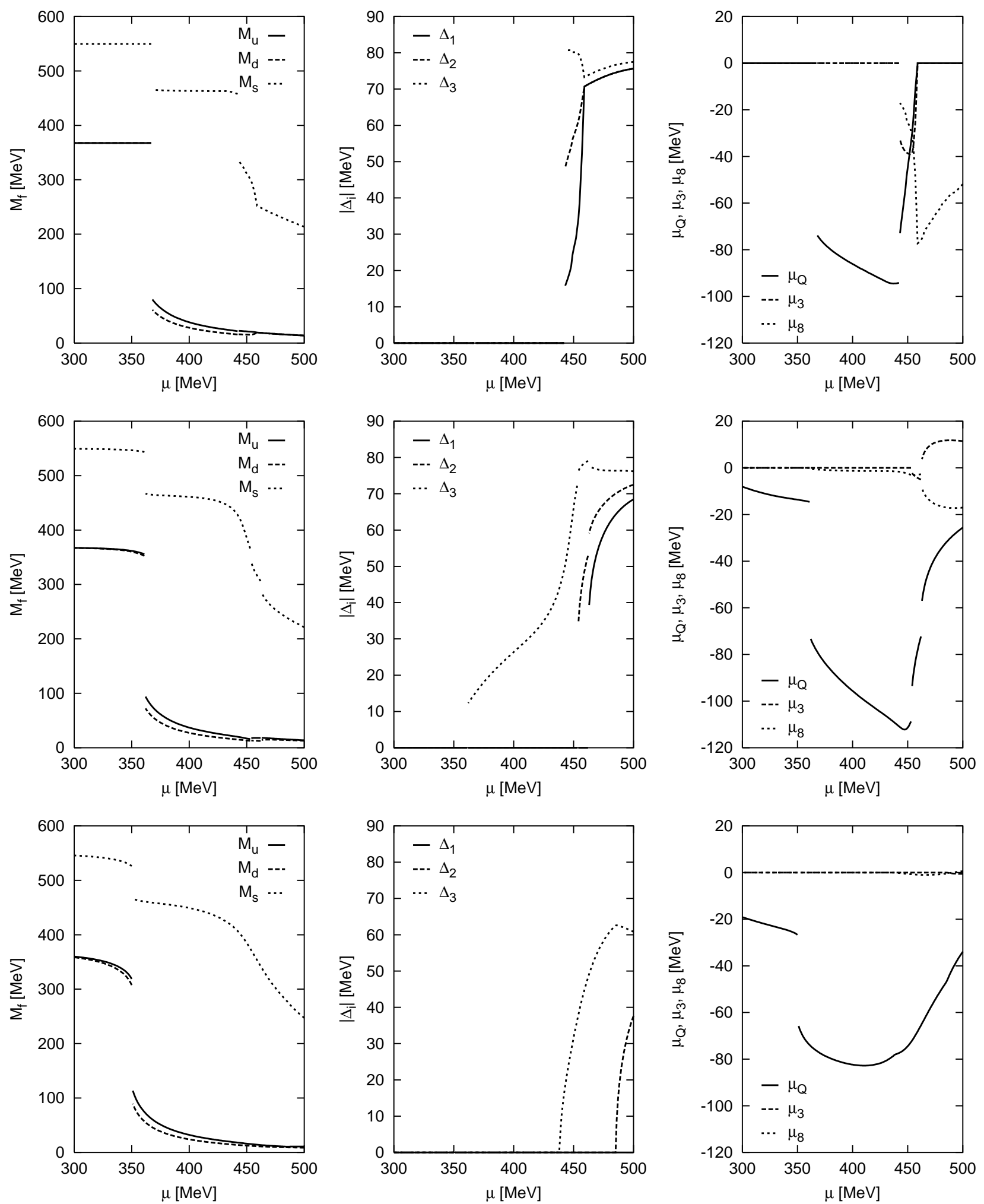

FIG. 3: Dependence of the quark masses, of the gap parameters, and of the electric and color charge chemical potentials on the quark chemical potential at a fixed temperature, $T=0 \mathrm{MeV}$ (three upper panels), $T=20 \mathrm{MeV}$ (three middle panels), and $T=40 \mathrm{MeV}$ (three lower panels). The diquark coupling strength is $G_{D}=\frac{3}{4} G_{S}$. 

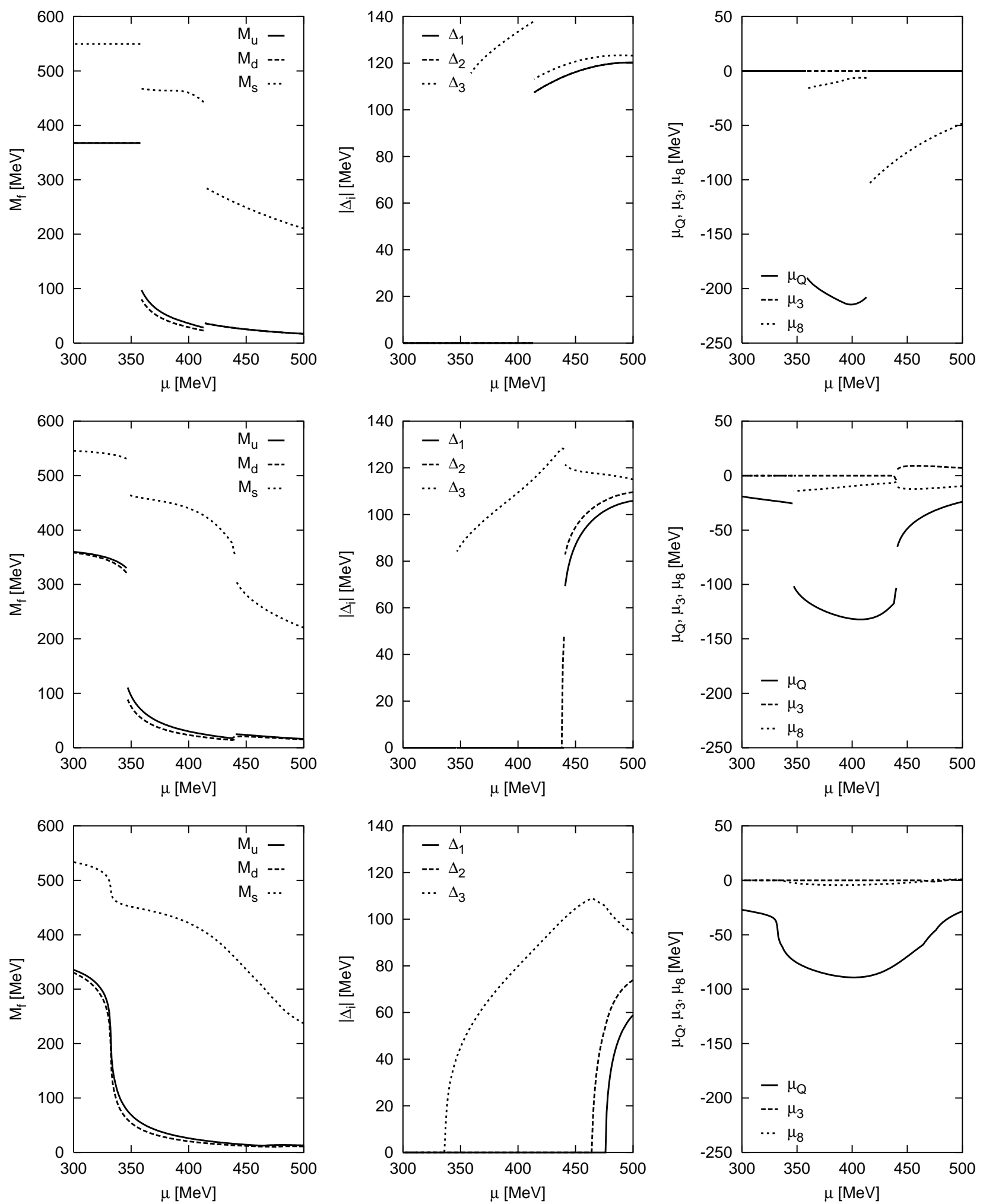

FIG. 4: The dependence of the quark masses, of the gap parameters and of the charge chemical potentials on the quark chemical potential at a fixed temperature, $T=0 \mathrm{MeV}$ (three upper panels), $T=40 \mathrm{MeV}$ (three middle panels), and $T=60 \mathrm{MeV}$ (three lower panels). The diquark coupling strength is $G_{D}=G_{S}$. 


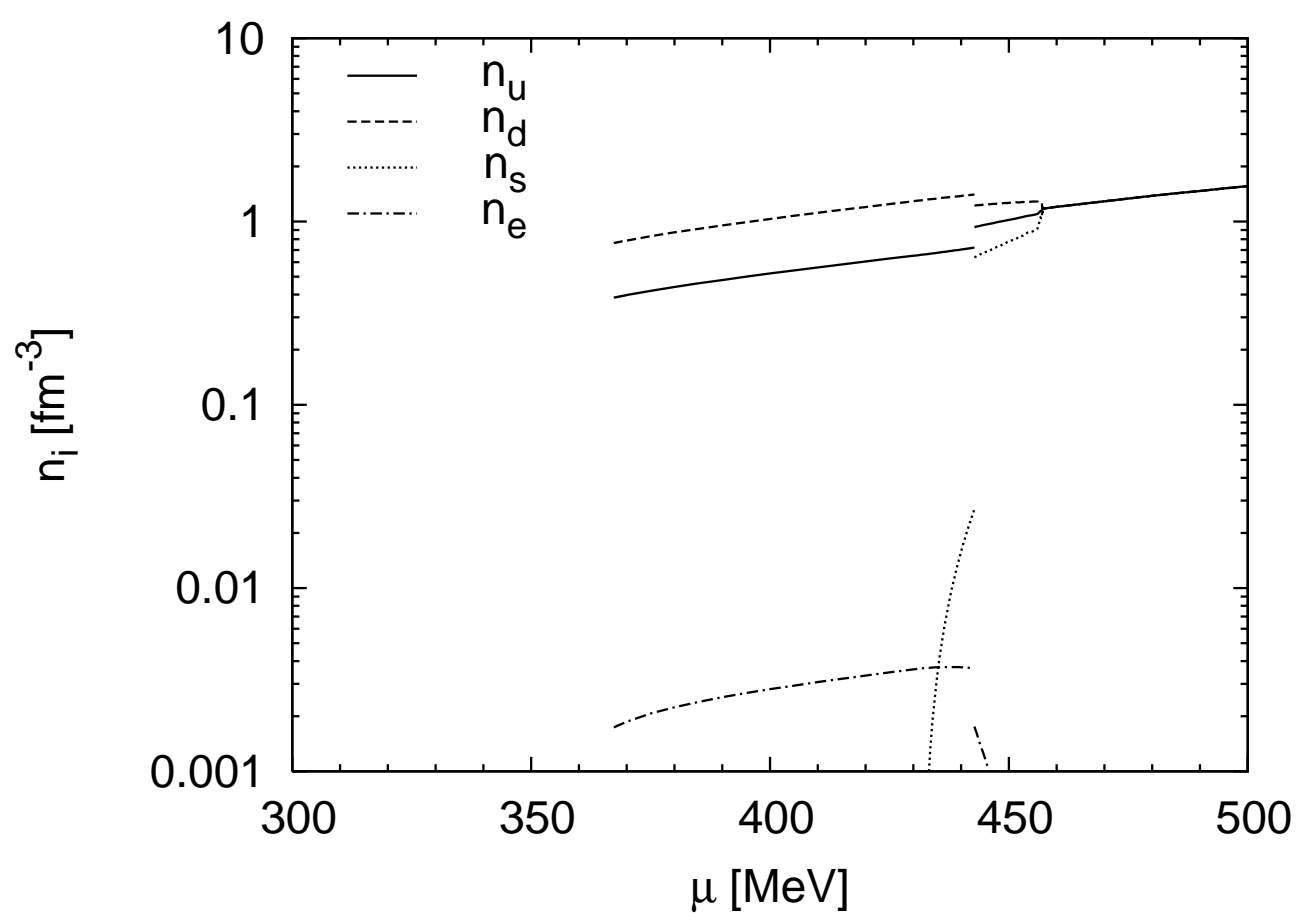

FIG. 5: The dependence of the number densities of quarks and electrons on the quark chemical potential at $T=0 \mathrm{MeV}$ for diquark coupling strength $G_{D}=\frac{3}{4} G_{S}$. Note that the densities of all three quark flavors coincide above $\mu=457 \mathrm{MeV}$. The density of muons vanishes for all values of $\mu$.

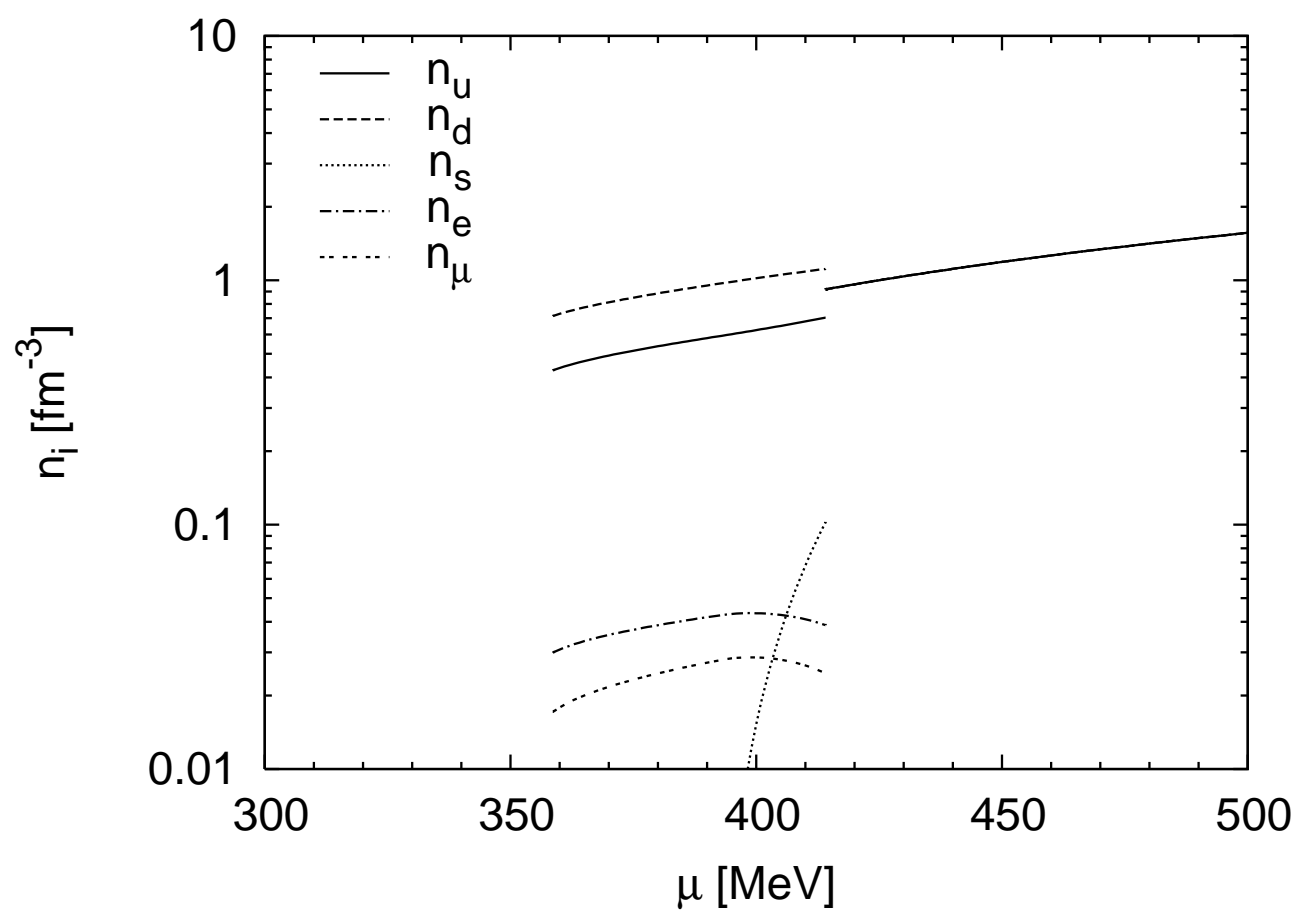

FIG. 6: The dependence of the number densities of quarks, electrons and muons on the quark chemical potential at $T=0 \mathrm{MeV}$ for diquark coupling strength $G_{D}=G_{S}$. Note that the densities of all three quark flavors coincide above $\mu=414 \mathrm{MeV}$. 


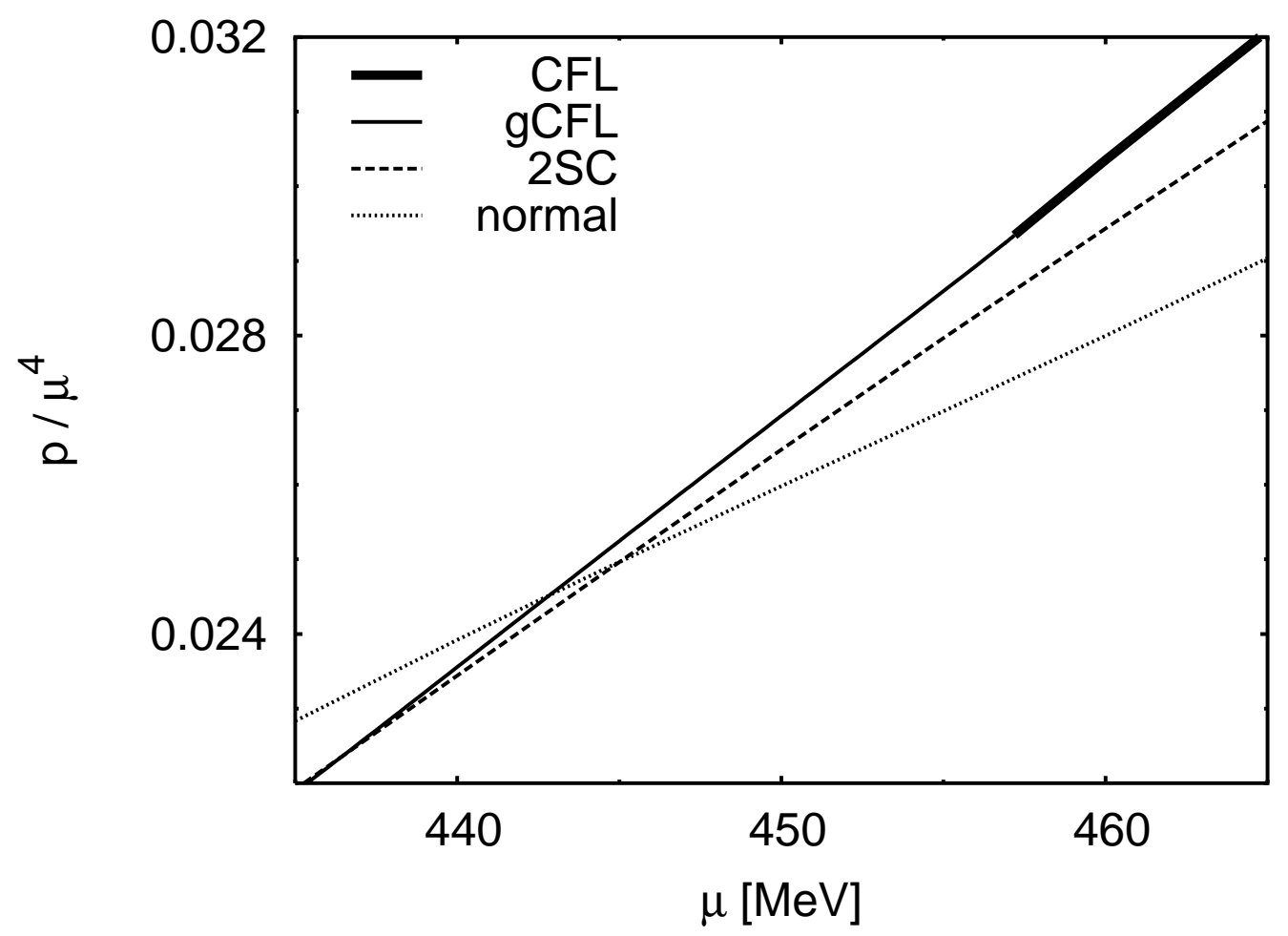

FIG. 7: Pressure divided by $\mu^{4}$ for different neutral solutions of the gap equations at $T=0$ as functions of the quark chemical potential $\mu$ : regular CFL (bold solid line), gapless CFL (thin solid line), 2SC (dashed line), normal (dotted line). The diquark coupling strength is $G_{D}=\frac{3}{4} G_{S}$. 